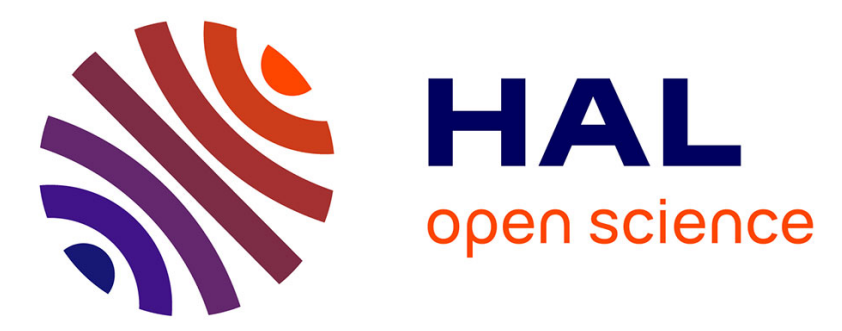

\title{
Numerical model of bone remodeling sensitive to loading frequency through a poroelastic behavior and internal fluid movements
}

Etienne Malachanne, David Dureisseix, Franck Jourdan

\section{- To cite this version:}

Etienne Malachanne, David Dureisseix, Franck Jourdan. Numerical model of bone remodeling sensitive to loading frequency through a poroelastic behavior and internal fluid movements. Journal of the mechanical behavior of biomedical materials, 2011, Bone remodeling, 4 (6), pp.849-857. 10.1016/j.jmbbm.2011.03.004 . hal-00595986

\section{HAL Id: hal-00595986 https://hal.science/hal-00595986}

Submitted on 22 Jun 2016

HAL is a multi-disciplinary open access archive for the deposit and dissemination of scientific research documents, whether they are published or not. The documents may come from teaching and research institutions in France or abroad, or from public or private research centers.
L'archive ouverte pluridisciplinaire HAL, est destinée au dépôt et à la diffusion de documents scientifiques de niveau recherche, publiés ou non, émanant des établissements d'enseignement et de recherche français ou étrangers, des laboratoires publics ou privés.

\section{(ㅇ)(1) $\$$}

Distributed under a Creative Commons Attribution - NonCommercial - NoDerivatives| 4.0 


\title{
Numerical model of bone remodeling sensitive to loading frequency through a poroelastic behavior and internal fluid movements
}

\author{
Etienne Malachanne ${ }^{1}$, David Dureisseix ${ }^{2}$ and Franck Jourdan ${ }^{3}$
}

${ }^{1}$ Centre des Matériaux de Grande Diffusion (CMGD), École des Mines d'Alès, 6 Avenue de Clavières, F-30319 Alès Cedex, France

${ }^{2}$ Laboratoire de Mécanique des Contacts et des Structures (LaMCoS), INSA Lyon / CNRS UMR 5259, 18-20, rue des Sciences, F-69621 Villeurbanne Cedex, France

${ }^{3}$ Laboratoire de Mécanique et Génie Civil (LMGC), University Montpellier 2 / CNRS UMR 5508, CC48, Place Eugène Bataillon, F-34095 Montpellier Cedex 5, France

\begin{abstract}
In this article, a phenomenological numerical model of bone remodeling is proposed. This model is based on the poroelasticity theory in order to take into account the effects of fluid movements in bone adaptation. Moreover, the proposed remodeling law stands from the classical 'Stanford' law, enriched in order to take into account the loading frequency, through fluid movements. This coupling is materialized by a quadratic function of Darcy velocity. The numerical model is carried out, using a finite element method, and calibrated using experimental results at macroscopic level, from the literature. First results concern cyclic loadings on a mouse ulna, at different frequencies between $1 \mathrm{~Hz}$ and $30 \mathrm{~Hz}$, for a force amplitude of $1.5 \mathrm{~N}$ and $2 \mathrm{~N}$. Experimental results exhibit a sensitivity to the loading frequency, with privileged frequency for bone remodeling between $5 \mathrm{~Hz}$ and $10 \mathrm{~Hz}$, for the force amplitude of $2 \mathrm{~N}$. For the force amplitude of $1.5 \mathrm{~N}$, no privileged frequencies for bone remodeling are highlighted. This tendency is reproduced by the proposed numerical computations. The model is identified on a single case (one frequency and one force amplitude) and validated on the other ones. The second experimental validation deals with a different loading regime: An internal fluid pressure at $20 \mathrm{~Hz}$ on a turkey ulna. The same framework is applied, and the numerical and experimental data are still matching in terms of gain in bone mass density.

This is a preprint of the publication: E. Malachanne, D. Dureisseix, F. Jourdan, Numerical model of bone remodeling sensitive to loading frequency through a poroelastic behavior and internal fluid movements, Journal of the Mechanical Behavior of Biomedical Materials 4:849-857, Elsevier, 2011. doi: 10.1016/j.jmbbm.2011.03.004, (C)2011 Elsevier. Licensed under the Creative Commons Attribution-NonCommercial-NoDerivatives 4.0 International http://creativecommons.org/licenses/bync-nd/4.0/
\end{abstract}

Keywords. biomechanics; tissue remodeling; diffusion; Darcy; finite elements; coupled problem

MSC 2010: $92 \mathrm{C} 10 ; 76 \mathrm{~S} 99 ; 74 \mathrm{~F} 10$

\section{Introduction}

First studies on bone remodeling were based on clinical observations, [40]. The first mathematical law describing this phenomenon, was a relationship between the strain engendered by external forces, and the variation of bone specific mass. Other remodeling laws were developed, in particular the one which will underpin this work, called in the following 'Stanford' law, [4, 3]. It describes the same phenomenon, relating this time a succession of daily loading conditions (via stresses) to a remodeling rate. Numerical studies, [11,39, 14], or theoretical development [1], using these models of bone remodeling were performed to predict bone adaptation. Apart from the coupling of the sole elasticity with remodeling, using Wolff law or a modified similar law or introducing several description scales, $[21,17,5]$, the influence of interstitial fluid on bone remodeling is often mentioned as in [9], or in [41] at cellular level. In such cases, various quantities can be selected as a remodeling primary variable (shear, pore pressure gradients...), as 
discussed in $[13,8,28,22,15,25]$ for instance. Other approaches rely on 'solid' quantities arising from additional constitutive mechanisms, such as micro-damage, see [29, 30, 23], with or without the influence of an interstitial fluid, as in [34,32], or viscosity in [2] that leads to a first order system, introducing a constitutive characteristic time. The present work also introduces a characteristic time, relying on hydraulic diffusion. Other couplings can be studied, such as thermoelectrical ones in [27], but this is out of the scope of this article. Moreover, [28] suggests that the loading frequency could have an influence on remodeling through internal fluid movements.

The aim of this article is to exemplify that a phenomenological macroscopic law can take into account the influence of the loading frequency through the fluid movement of a poroelastic model for bone remodeling. Following the works of $[4,3]$, the proposed model is not based on micro-mechanism at cellular or tissue levels, but nevertheless leads to a predictive numerical model once identified for a given bone micro-structure.

As a first step the poroelastic model will be recalled, to represent the presence of both fluid and solid phases. In a second step, a remodeling law will be developed, and numerical results obtained with this law will be compared to experimental data from the literature.

\section{Methods and Procedures}

\subsection{Poroelastic model}

The poroelasticity theory describes interactions between solid and fluid phases, at a macroscopic level, in a porous media like bone. Its main equations are detailed in $[7,6]$, especially for the conservation equations that are not recalled here. Boundary conditions will be detailed during the numerical identification of the remodeling law.

For a linear poroelastic model, using Einstein's notation, the relationship between total stress tensor $\sigma_{i j}(i, j \in\{1,2,3\})$, strain tensor $\varepsilon_{i j}$ and pore pressure $p$ is

$$
\sigma_{i j}=D_{i j k l} \varepsilon_{k l}-\alpha p \delta_{i j}
$$

where $\delta_{i j}$ is the Kronecker symbol $\left(\delta_{i j}=1\right.$ if $i=j$, and 0 otherwise $)$ and $\alpha$ is the Biot coefficient. In this study an isotropic poroelastic model was chosen, as in [7]. Anisotropic elastic parameters are now available for bone, see for instance [38], but less has been done for a poroelastic behaviour. Using an anisotropic model may change a little bit the values of the identified parameters for remodeling since we use a numerical computation for identification, but not the major effects of the remodeling. Herein, we still rely on an isotropic model. In this case, the fourth-order Hooke elasticity tensor $D_{i j k l}$ of the drained material depends only on two parameters, for instance: Young modulus $E$ and Poisson coefficient $\nu$. Moreover, the relationship between fluid accumulation $q$, pore pressure $p$ and relative volume variation, is

$$
q=\frac{1}{Q} \dot{p}+\alpha \dot{\varepsilon}_{k k}
$$

$Q$ is Biot modulus, taking compressibility into account.

Finally, the Darcy law relates the opposite of Darcy velocity $W_{i}$ and pore pressure gradient $Z_{i}=p_{, i}$ :

$$
W_{i}=H Z_{i}
$$

For an isotropic behaviour, $H=k / \mu$, where $k$ is the intrinsic permeability and $\mu$ the dynamic viscosity of the interstitial fluid. Table 1 gives these material parameters for cortical bone; apart from permeability $H$ which was determined by a coupled experimental and numerical method in [18], the other values are reported from [33].

At least two time scales arise from this modeling. Indeed, disregarding structural effects on a stress driven monodimensional poroelastic problem leads to, following [24],

$$
p_{, 11}=\frac{\tau}{L^{2}} \dot{p}+\frac{\alpha}{E} \dot{\sigma}_{11}
$$

with an intrinsic time for the poroelastic model

$$
\tau=L^{2} \frac{1}{H}\left(\frac{1}{Q}+\frac{\alpha^{2}}{E}\right)
$$

where $L$ is a characteristic length of the structure, and a time scale arising from the loading $\dot{\sigma}_{11}(t)$. 


\begin{tabular}{ll}
\hline Young modulus & $E=15 \mathrm{GPa}$ \\
Poisson ratio & $\nu=0.325$ \\
Biot modulus & $Q=15 \mathrm{GPa}$ \\
Biot coefficient & $\alpha=0.78$ \\
Hydraulic permeability & $H=1.1 \times 10^{-13} \mathrm{~m}^{4} \mathrm{~N}^{-1} \mathrm{~s}^{-1}$ \\
\hline
\end{tabular}

Table 1: Isotropic poroelastic material parameters

\subsection{Remodeling law}

\subsubsection{The classical 'Stanford' law}

The 'Stanford' law, proposed in [4, 3], describes the bone adaptation at macroscopic scale, relating a set of stresses corresponding to successive loading conditions, on a daily basis, to the remodeling rate. A daily stress stimulus, similar to a cumulative law, is defined as

$$
\psi=\left(\sum_{i} n^{(i)}\left(\bar{\sigma}^{(i)}\right)^{m}\right)^{1 / m}
$$

$n^{(i)}$ is the number of cycles of load type $i$ per day, and $m$ is an empirical constant. $\bar{\sigma}^{(i)}$ is an average of the local stress (at each point $M$ ) on the loading type time interval $I^{(i)}$, of length $T^{(i)}$, during which load type $i$ is prescribed to the structure:

$$
\bar{\sigma}^{(i)}(M)=\int_{I^{(i)}} \sigma_{\mathrm{eq}}(M, t) \frac{d t}{T^{(i)}}
$$

$\sigma_{\text {eq }}$ is here the von Mises equivalent stress (therefore we assume that hydrostatic pressure has no influence on remodeling, unlike shear stresses). In practise, this average can be approximated by simulating only one cycle of loading, assuming that the stabilized cycle does not largely differ from this.

At a microscopic scale, there are privileged material surfaces where bone remodeling takes place, see $[26,20,4]$. The 'specific surface', [20], $S_{v}$, is related to the bone surface available as remodeling site in a bone sample, divided by the bulk volume of the sample. Indeed, it is a function of bone specific mass. In $[20,4]$, an interpolation by a fifth-degree polynomial function is suggested, for which an identification leads to

$$
S_{v}(\rho)=-0.3005 \rho^{5}-0.0455 \rho^{4}+1.9722 \rho^{3}-4.5903 \rho^{2}+6.7334 \rho-0.0115
$$

when $S_{v}$ is expressed in $\mathrm{mm}^{2} / \mathrm{mm}^{3}$ and $\rho$ in $\mathrm{g} / \mathrm{cm}^{3}$ (see Figure 1). A remodeling rate $\dot{r}$ can be now introduced, relating the bone specific surface $S_{v}$, to the bone specific mass rate $\dot{\rho}$ :

$$
\dot{\rho}=\dot{r} \rho_{v} S_{v}(\rho)
$$

$\rho_{v}$ is the true specific mass of the bone tissue (the fully mineralized solid phase), which is an additional material parameter in the model.

The remodeling rate is finally related to the difference between the daily stress stimulus $\psi$ and a reference daily stress stimulus $\psi_{\text {ref. }}$ With values close to $\psi_{\text {ref }}$, remodeling does not occur and macroscopic bone specific mass is constant; this is the so-called dead zone. Apposition and resorption may occur, depending on the relative value of the remodeling stimulus (see Figure 2):

$$
\dot{r}=\left\{\begin{array}{lll}
c\left(\psi-\psi_{\text {ref }}\right)+c w & \text { if } \quad \psi-\psi_{\text {ref }}<-w \\
c\left(\psi-\psi_{\text {ref }}\right)-c w & \text { if } \quad \psi-\psi_{\text {ref }}>w \\
0 & \text { if }-w<\psi-\psi_{\text {ref }}<w
\end{array}\right.
$$

Constants $c, w$ (half width of the dead zone) and the reference daily stress stimulus $\psi_{\text {ref }}$ are material macroscopic coefficients that depend on bone properties. As in [3], $w$ is assumed to be directly related to $\psi_{\text {ref }}$ with $w=10 \% \times \psi_{\text {ref }}$, and $c$ is determined by assuming that a daily stress stimulus $\psi=2 \psi_{\text {ref }}$ would correspond to $\dot{r}=1 \mu \mathrm{m} /$ day. With these assumptions, the material parameters of the remodeling law, $c$ and $w$, now only depend on a single parameter $\psi_{\text {ref }}$ that remains to be determined, which will be done in the following. 


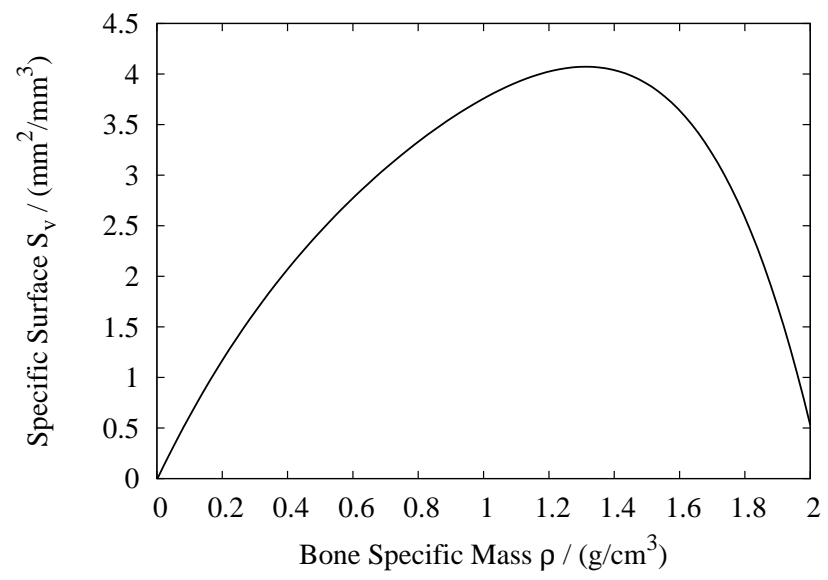

Figure 1: Specific surface vs specific mass (after [20])

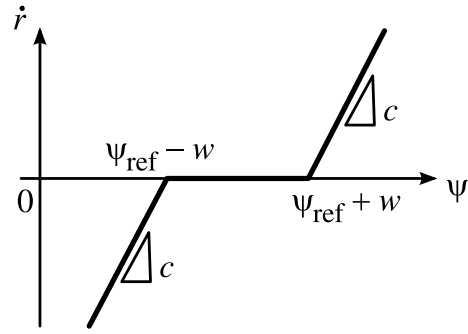

Figure 2: Remodeling rate vs daily stress stimulus. We can see a dead zone where bone specific is constant 


\subsubsection{Remodeling law taking into account loading frequency}

The objective is to built a numerical model of bone remodeling taking into account internal fluid movements and loading frequency, to reproduce experimental results of the literature. For the 'Stanford' remodeling law, both the stress and the number of loading cycles are considered and weighted by the empirical constant $m$. This law does not take into account the frequency of each loading type, though some experiments have shown its influence on bone remodeling, such as [37, 35, 10].

In this article, we propose first to introduce a scaling parameter in the daily stress stimulus: the time fraction of each load type $i, T^{(i)} / T$, where $T^{(i)}$ is the duration of load type $i$ at frequency $f^{(i)}$ within each day, and $T$ is equal to one day. Second, we assume that the loading frequency effects could be taken into account via bone internal fluid movements. Indeed, considering (1), the loading frequency has an influence on fluid quantity evolutions; we propose here to use the Darcy velocity as a mechanism to influence bone adaptation (this choice refers to the assumption of cell sensitivity to shearing at microscopic scale, see $[8,12])$. Thus the number of cycles is replaced in 'Stanford' law by an adimensional function of Darcy velocity, $g\left(\bar{w}^{(i)}\right)$, with the average of velocity norm:

$$
\bar{w}^{(i)}=\int_{I^{(i)}} \sqrt{W_{k}^{(i)} W_{k}^{(i)}} \frac{d t}{T^{(i)}}
$$

For the scope of this article, the function $g$ is purely phenomenological, and is not obtained from microbiomechanical mechanisms in a microscale analysis, such as migration and cellular differentiation, see for instance $[16,1]$. Therefore, the simpler function, allowing the experimental results to be recovered, is sought for. Finally, empirical constant $m$ is removed from the daily stress stimulus, that is now expressed as

$$
\psi=\sum_{i} \frac{T^{(i)}}{T} \bar{\sigma}^{(i)} g\left(\bar{w}^{(i)}\right)
$$

The daily stress stimulus $\psi$ is still homogeneous to a pressure. The reference daily stress stimulus $\psi_{\text {ref }}$ can be obtained with the same expression as (8), considering a loading condition that maintains bone specific mass constant (more precisely, in the center of the dead zone). Thus, $\psi_{\text {ref }}$, which is still considered as a material parameter, is obtained such as

$$
\psi_{\text {ref }}=\frac{T_{\text {ref }}}{T} \bar{\sigma}_{\text {ref }} g\left(\bar{w}_{\text {ref }}\right)
$$

with a single loading type whose characteristics are a time fraction $T_{\text {ref }} / T$, an average stress amplitude $\bar{\sigma}_{\text {ref }}$, and an average Darcy velocity amplitude $\bar{w}_{\text {ref }}$. As soon as this reference loading is defined and the function $g$ selected, the reference daily stress stimulus can be obtained. To access local stress and Darcy velocity, a numerical poroelastic simulation is nevertheless required, with previously identified poroelastic parameters. Note that this definition suggests that a uniform stress and a uniform Darcy velocity are obtained on a sample. Since the only available informations are related to global bone structures, a spatial averaging is also required to get the final value of $\psi_{\text {ref }}$.

\section{Results}

To complete the model, the material parameter $\psi_{\text {ref }}$ and the function $g(\bar{w})$ need to be identified. To do so, experimental data from the literature are used, and the model is validated by comparing its results to other experimental results. A first experimental study, from [37], for which the influence of loading frequency and internal fluid movements on bone remodeling has been highlighted, is used as a first identification and validation of the proposed model, on a mouse ulna. A second study, from [28], for which an internal pressure is prescribed on a turkey ulna, is used as a second example and validation.

\subsection{Structural computation for several loading frequencies}

\subsubsection{Experimental study from literature}

In [37]. the authors propose a method to measure the remodeling rate, that was called 'Bone Formation Rate' and expressed in $\mu \mathrm{m}^{3} \mu \mathrm{m}^{-2}$ year $^{-1}$, after different cyclic loading frequencies. The considered frequencies are $1 \mathrm{~Hz}, 5 \mathrm{~Hz} 10 \mathrm{~Hz}, 20 \mathrm{~Hz}, 30 \mathrm{~Hz}$ (lower values can been found in other investigations, e.g. $[35,10])$ and their aim was to determine the most efficient frequency for bone remodeling. Tests, depicted 
in Figure 3-A, were conducted in [31, 37] on mice ulna, with cyclic global traction/compression loadings. 120 loading cycles were performed per day on a test for each of the selected frequencies, with force amplitudes of $1.5 \mathrm{~N}$ and $2 \mathrm{~N}$. Since the number of cycles was constant, the duration of each loading decreases when increasing frequency. These loadings were performed during three days, and mice were killed 18 days after the first loading.
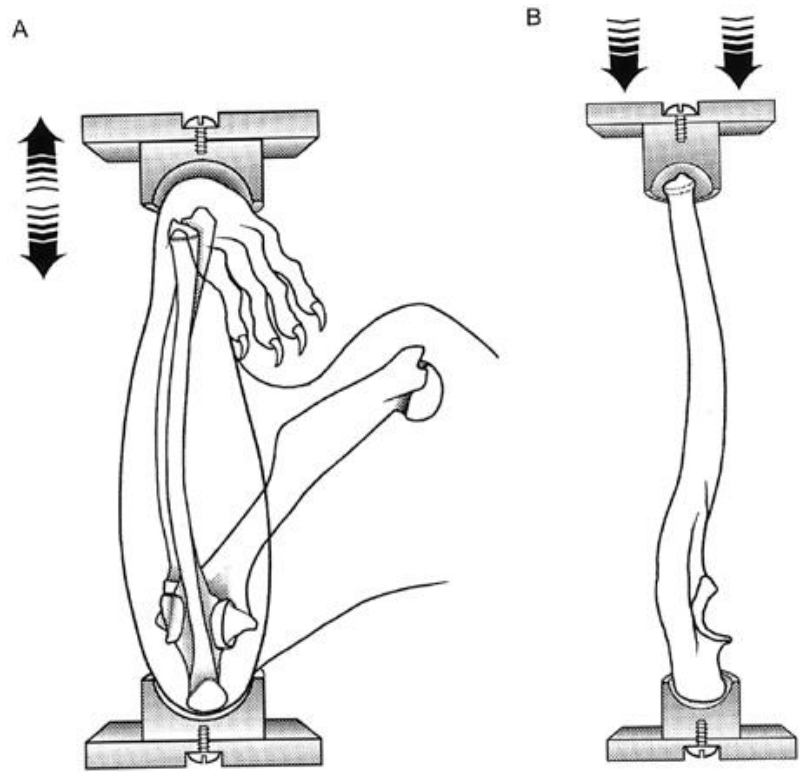

Figure 3: Mice ulna under cyclic loading (after [31]). Reproduced from J Bone Miner Res 2002; 17; 1545-1554 with permission of Wiley and the American Society for Bone and Mineral Research

Experimental results are represented in Figures 4 and 5. These results exemplify the effect on bone remodeling of the loading frequency. This effect is not monotonous with the frequency value. If there is not a clear tendency for the loading amplitude of $1.5 \mathrm{~N}$, for the case of an amplitude of $2 \mathrm{~N}$, the most efficient values for bone remodeling are between $5 \mathrm{~Hz}$ and $10 \mathrm{~Hz}$, and the remodeling rate decreases after $10 \mathrm{~Hz}$.

\subsubsection{Numerical results and comparisons}

In this section we propose first a phenomenological identification of the daily stress stimulus. Then the chosen remodeling law is validated with the experimental results of [37]. To this end, the discretisation of the poroelastic model with remodeling, presented in the previous sections, is performed using a finite element method with hybrid elements for displacement and pressure interpolations (P2 with straight edges / continuous P1), and an implicit Euler time integration scheme.

Ulna have a length of about $2 \mathrm{~cm}$, inner diameter of $2 \mathrm{~mm}$ and outer diameter of $3 \mathrm{~mm}$, and a threedimensional mesh of the mouse ulna is carried out with the finite element code Cast3M, [36], Figure 7, and the boundary conditions will be selected as close as possible to the experimental situation. In the following, the upper end section of the ulna is denoted with $\partial_{1} \Omega$ and the lower one with $\partial_{2} \Omega$.

\subsubsection{Choice of a reference daily stress stimulus}

The poroelastic material parameters are selected in Table 1. Concerning the remodeling material parameters, using the relationships of section 2.2.1, reduces the number of unknowns to the single parameter $\psi_{\text {ref }}$ as soon as a reference loading is found. Such a loading is considered as a daily condition maintaining bone mass constant, i.e. a 'standard' physical activity, for a $55 \mathrm{~g}$ mouse. On the upper section $\partial_{1} \Omega$, normal stress and pore pressure are prescribed, according to the mouse weight, to $2.5 \times 10^{4} \mathrm{~Pa}$. On the lower section $\partial_{2} \Omega$, nil displacement and fluid flow are prescribed. Lateral surfaces are free of forces, with nil fluid flow. Considering a $10 \mathrm{~h}$ duration of awareness per day $\left(T_{\text {ref }} / T=10 / 24\right)$, a numerical simulation is performed. For this low frequency case, the Darcy velocity is small and can be neglected; therefore, for this loading case, the approximation $g\left(\bar{w}_{\text {ref }}\right) \approx g(0)=1$ is used. Finally, $\psi_{\text {ref }}=\frac{T_{\text {ref }}}{T} \bar{\sigma}_{\text {ref }}$ is obtained 


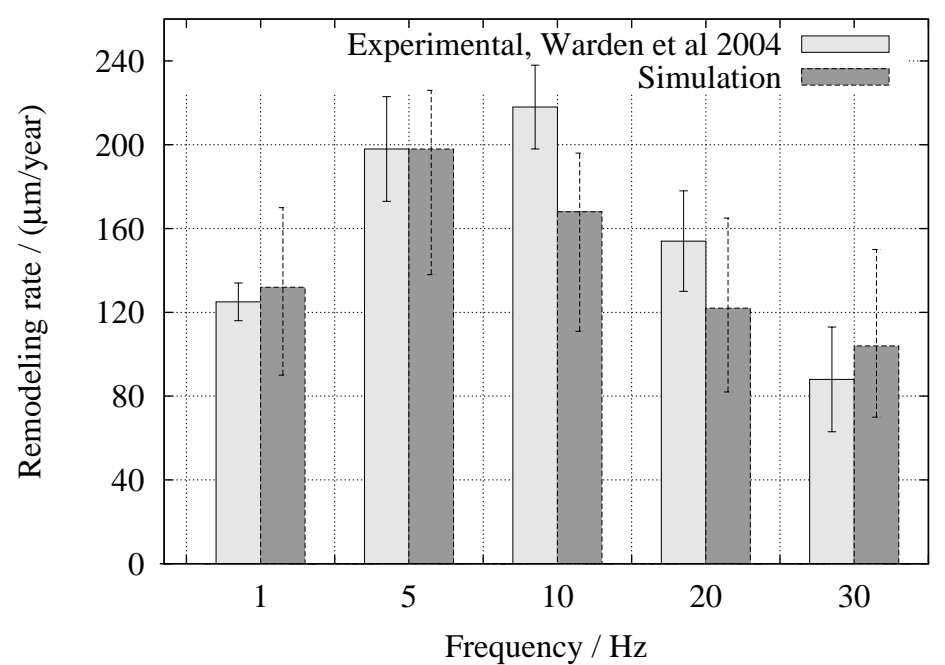

Figure 4: Remodeling rate vs loading frequency, for a loading amplitude of $2 \mathrm{~N}$. Parameter identification is performed for the sole frequency of $1 \mathrm{~Hz}$, and the model is compared to the experimental values for the other frequencies. Error bars on experimental data are related to dispersions, error bars on numerical data are related to sensitivity analysis on parameter $\psi_{\text {ref }}$ (tested for a relative variation of $\pm 10 \%$ )

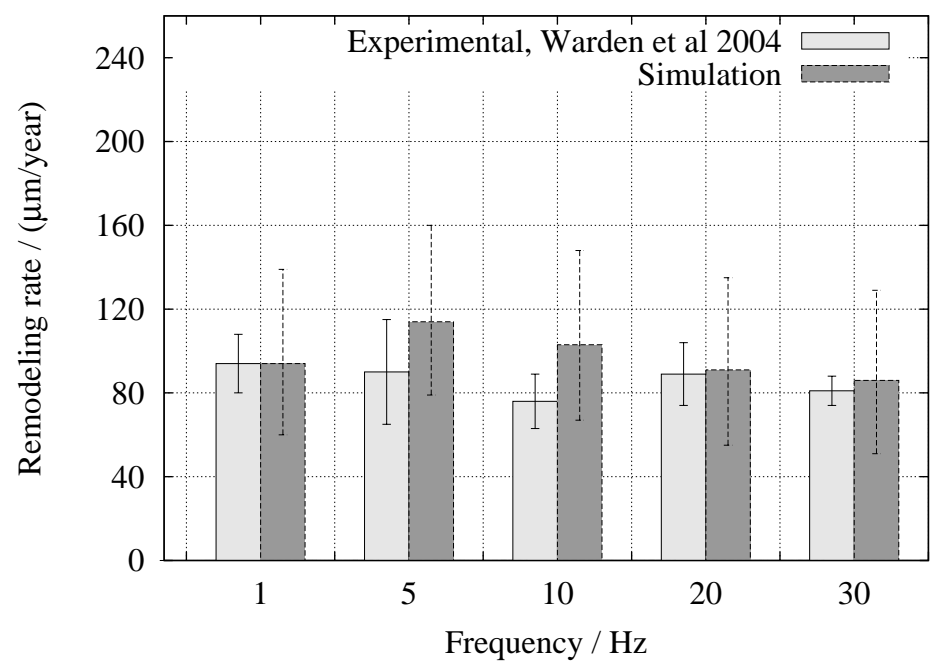

Figure 5: Remodeling rate vs loading frequency. Validation for a loading amplitude of $1.5 \mathrm{~N}$. One can notice that numerical results still agree with experimental results, though no identification is performed. Error bars on experimental data are related to dispersions, error bars on numerical data are related to sensitivity analysis on parameter $\psi_{\text {ref }}$ (tested for a relative variation of $\pm 10 \%$ ) 


\begin{tabular}{ll}
\hline$\rho_{v}$ & $1.96 \mathrm{~g} / \mathrm{cm}^{3}$ \\
$\psi_{\text {ref }}$ & $1.07 \times 10^{4} \mathrm{~Pa}$ \\
$w$ & $1.07 \times 10^{3} \mathrm{~Pa}$ \\
$c$ & $1.04 \times 10^{-4} \mu \mathrm{m} . \mathrm{Pa}^{-1}$. day $^{-1}$ \\
\hline
\end{tabular}

Table 2: Remodeling material parameters

from a first simulation for the reference case, with a spatial averaging to get a unique value for $\psi_{\text {ref }}$ The remaining material parameters $c$ and $w$ are deduced from this value, and are reported in Table 2.

\subsubsection{Identification and validation of function $g$}

The aim of this section is to identify a suitable function $g$ to reproduce experimental data. The new boundary conditions now consist in the following: on the upper section $\partial_{1} \Omega$, a cyclic normal stress, and a pore pressure are prescribed as $F_{0} \sin (\omega t)$. The pulsation depends on the loading frequency: $\omega^{(i)}=2 \pi f^{(i)}$. For the identification of the function $g$, the case with a force amplitude $A=2 \mathrm{~N}$ was used. The upper surface being $S=2.22 \times 10^{-5} \mathrm{~m}^{2}$, the external pressure is

$$
F_{0}=\frac{A}{S}=9 \times 10^{4} \mathrm{~Pa}
$$

On the lower section $\partial_{2} \Omega$, nil displacement and fluid flow are prescribed. For lateral surfaces, the boundary conditions are still the same. Figure 6 shows the average Darcy velocity $\bar{w}^{(i)}$ versus the loading frequency $f^{(i)}$, at a representative integration point. Though it increases with the frequency, this evolution is not linear.

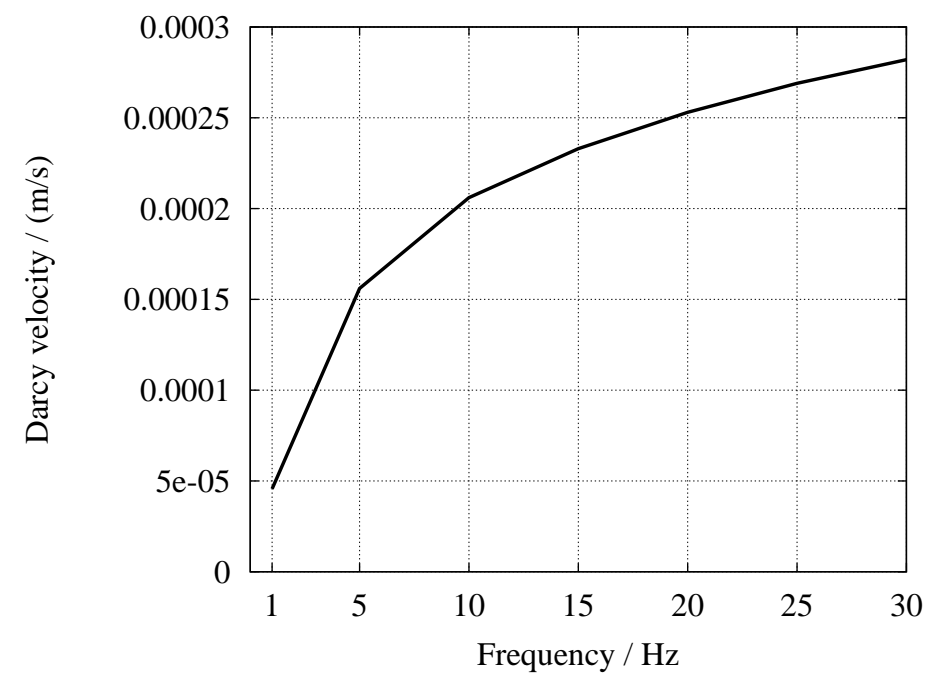

Figure 6: Simulated values of Darcy velocity for a poroelastic model vs loading frequency. One can notice that the slope decreases with the frequency.

The proposed phenomenological function $g$ is

$$
g(\bar{w})=1+a^{2} \bar{w}^{2}
$$

where $a$ is a new material parameter, depending on bone nature. It is identified numerically for the first frequency of $1 \mathrm{~Hz}$ : for several values of this parameter, the average remodeling rate is numerically computed and reported in Table 3. According to experimental results, for a loading frequency of $1 \mathrm{~Hz}$, the remodeling rate is equal to $\dot{r}=120 \mu \mathrm{m} /$ year. Thus $a$ is taken equal to $a=1.5 \times 10^{5} \mathrm{~s} . \mathrm{m}^{-1}$.

With this value of parameter $a$, the remodeling rate $\dot{r}$ is numerically computed for the other frequencies, as shown in Figure 4. The agreement is satisfactory. Using this function, the remodeling rate can now also be computed with a loading amplitude $A=1.5 \mathrm{~N}$ : Numerical results are provided in Figure 5 , for which the correlation is also satisfactory. 


\begin{tabular}{cc}
\hline$a /$ s.m $^{-1}$ & $\dot{r} / \mu \mathrm{m}$. year $^{-1}$ \\
\hline $10^{2}$ & 52 \\
$10^{3}$ & 53 \\
$10^{4}$ & 53 \\
$10^{5}$ & 82 \\
$1.5 \times 10^{5}$ & 118 \\
\hline
\end{tabular}

Table 3: Remodeling rate $\dot{r}$ obtained with simulation for several values of the material parameter $a$, and for a loading frequency $f=1 \mathrm{~Hz}$

At a first time, a simple linear function of Darcy velocity was chosen for $g$, [19]. In this case, the influence of Darcy velocity was not sufficient to reproduce experimental results. Because of a lack of correlation between experimental and numerical results, this linear function $g$ was discarded in favor of a quadratic function (11).

The aim of this work is also to propose a predictive numerical model for bone remodeling. Indeed, the simulation, using a 3D mesh of mouse ulna, distinguishes the areas where the remodeling is the largest. The evolution of bone specific mass is shown in Figure 7, for the different frequencies.
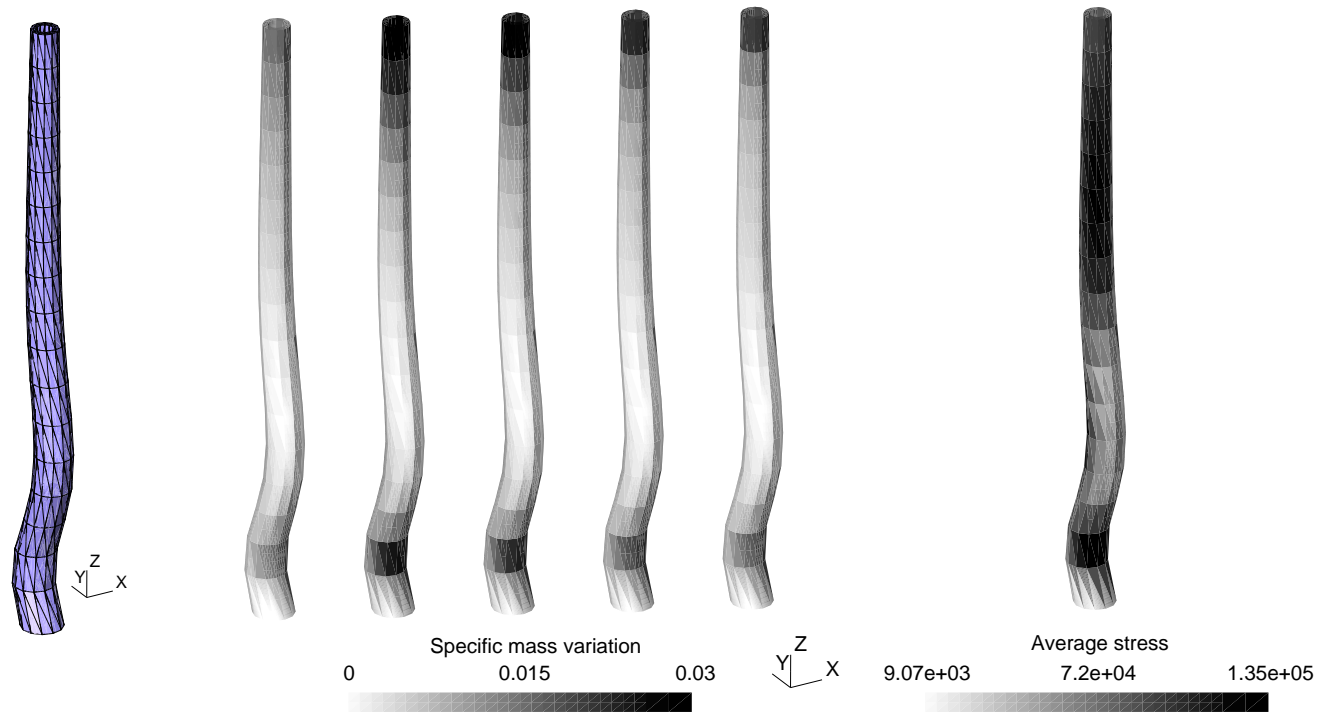

Figure 7: On the left: mesh of mouse ulna. In the center: gain in bone specific mass on mouse ulna, for several loading frequency (from left to right: $f=1 \mathrm{~Hz}, 5 \mathrm{~Hz}, 10 \mathrm{~Hz}, 20 \mathrm{~Hz}, 30 \mathrm{~Hz}$ ). On the right: mean stress solution $\bar{\sigma}$ for a loading amplitude of $2 \mathrm{~N}$.

\subsection{Validation on a low amplitude and high frequency loading}

\subsubsection{Experimental procedure from literature}

Another experimental study had shown the importance of fluid movement, and internal pressure, for bone remodeling, in [28]. In this work, the authors applied a fluid stimulus on a turkey ulna, with an internal cyclic high frequency pressure, $20 \mathrm{~Hz}$, and a low amplitude, $8000 \mathrm{~Pa}$, see Figure 8 . This loading was applied $10 \mathrm{~min}$ per day, during 28 days. At the end of this procedure, turkey were killed and loaded ulna were cut out and gain in bone mass density were measured and compared with reference turkeys. The main conclusion of this work was the significant mean bone mass increase, with an average of $18 \%$, for turkeys submitted to cyclic loading.

\subsubsection{Numerical results and comparison}

A numerical simulation is carried out, using the model presented in previous sections. Poroelastic parameters are the same as previously numerical computation, selected in Table 1. 


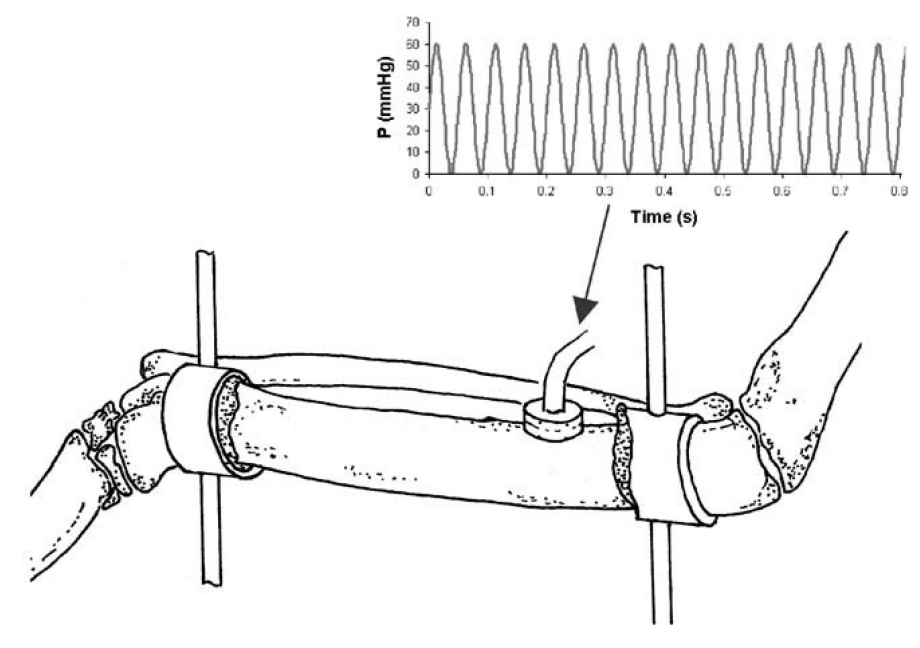

Figure 8: Turkey ulna under internal pressure, experimental procedure from [28]. Reproduced from Journal of Biomechanics 36:1427-1437, copyright Elsevier (2003) with permission

\begin{tabular}{ll}
\hline$\rho_{v}$ & $1.96 \mathrm{~g} / \mathrm{cm}^{3}$ \\
$\psi_{\text {ref }}$ & $3.6 \times 10^{3} \mathrm{~Pa}$ \\
$w$ & $3.6 \times 10^{2} \mathrm{~Pa}$ \\
$c$ & $2.7 \times 10^{-10} \mu \mathrm{m} . \mathrm{Pa}^{-1} . \mathrm{day}^{-1}$ \\
\hline
\end{tabular}

Table 4: Remodeling material parameters for turkey ulna

An axisymetric mesh of the turkey ulna is carried out, as shown in Figure 9, and boundary conditions are selected as close as possible to the experimental situation. An internal pressure is applied as $F_{0} \sin (\omega t)$, with $\omega=2 \pi f$, where $f$ is the loading frequency of $20 \mathrm{~Hz}$ and $F_{0}$ is the amplitude of $8000 \mathrm{~Pa}$. On external surfaces, a nil fluid flow is applied.

Figure 9: Axisymetric mesh of half of the Turkey ulna

Two material parameters have to be identified for this new experimental case. The reference daily stress stimulus $\psi_{\text {ref }}$ is calculated considering a physical activity useful to maintain bone mass density for a turkey ulna, here $\psi_{\text {ref }}=3.6 \times 10^{3} \mathrm{~Pa}$. As previously, the other parameters $c$ and $w$, are related to this reference value, and are given in Table 4 .

Material parameter $a$ is calibrated with the same procedure used in previous section. Here cyclic loading is only performed for a frequency of $20 \mathrm{~Hz}$, and $a$ has to be found in order to obtain a mean gain in bone mass density, around 18\%, agreeing with experimental results of [28]. Several values of parameter are tested and gain in bone mass density after 28 days of loading is numerically computed as

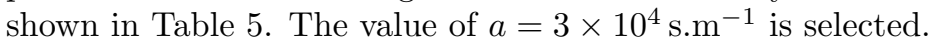

The local gain in specific bone mass density on turkey ulna is depicted in the field of Figure 10 and values range from $0 \%$ to $32 \%$, which agrees with the gain of $30 \%$ on periosteal surface, announced in $[28]$.

\section{Discussion}

The theoretical and numerical macroscopic model of bone remodeling, presented in previous sections, takes into account interactions between fluid and solid phases of a poroelastic medium, and the remodeling 


\begin{tabular}{ll}
\hline$a / \mathrm{s.m}^{-1}$ & $\begin{array}{l}\text { Mean gain in bone mass density } \\
\text { after 28 days of loading }\end{array}$ \\
\hline $0.1 \times 10^{4}$ & $0 \%$ \\
$1.5 \times 10^{4}$ & $3 \%$ \\
$2 \times 10^{4}$ & $6 \%$ \\
$2.5 \times 10^{4}$ & $11 \%$ \\
$3 \times 10^{4}$ & $18 \%$ \\
$3.5 \times 10^{4}$ & $20 \%$ \\
\hline
\end{tabular}

Table 5: Simulated mean gain in bone mass density on turkey ulna, with respect to several values for the parameter $a$

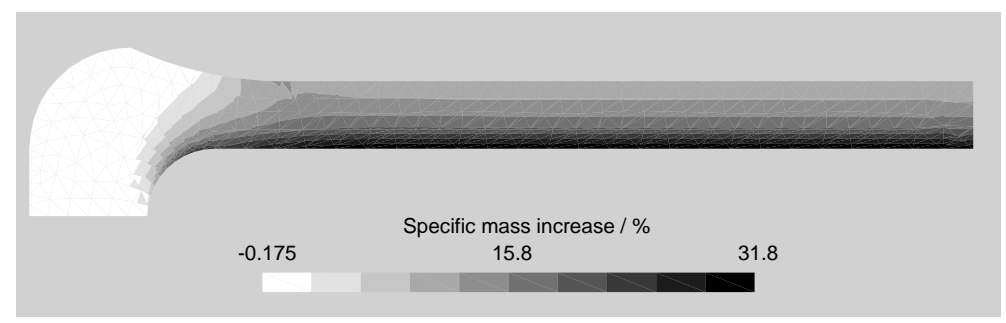

Figure 10: Distribution of local specific bone mass density, after 28 days of loading

stimulus. The trends given by numerical results agree with experimental results which exhibit sensitivity to the loading frequency through the fluid movements. This is taken into account in the model, using a quadratic function of Darcy velocity (11).

The numerical model reproduces privileged loading frequencies for bone remodeling, as for the experimental study, when the amplitude of loading is of $2 \mathrm{~N}$ : The most important increase in remodeling rate occurs close to $5 \mathrm{~Hz}$. After $10 \mathrm{~Hz}$, the gain in bone density continues to decrease. Classical remodeling laws, defined at macroscopic scale, like Wolff law in [40] and 'Stanford' law in [4, 3], do not allow this kind of phenomenon to be reproduced. Indeed these laws fail to take loading frequency into account. 'Stanford' law considers only the number of loading cycles, which is constant for this study, whereas the proposed model depends also on a time fraction, $T^{(i)} / T$, which decreases when the loading frequency increases.

The second cyclic loading on the mouse ulna, with a force amplitude of $1.5 \mathrm{~N}$, provides once again numerical results similar to experiments, without additional identification. Between $5 \mathrm{~Hz}$ and $10 \mathrm{~Hz}$, values for a loading at $1.5 \mathrm{~N}$ are roughly half of those obtained for a force amplitude of $2 \mathrm{~N}$ and do not clearly exhibit a privileged frequency for bone remodeling. This difference between these two results could be explained by a low value of the remodeling stimulus $\psi$.

An other numerical computation is carried out, based on a different experimental study in [28]. In this case, a low amplitude $(8000 \mathrm{~Pa})$ and a high frequency loading $(20 \mathrm{~Hz})$ is applied on a turkey ulna during 28 days, providing an increase in bone mass density of $18 \%$. Two material parameters have been calibrated to reproduce experimental results, parameter $a$ and the reference daily stress stimulus $\psi_{\text {ref. }}$. Numerical results had provided a significant increase in bone mass density, as in the experimental study, in spite of a low amplitude loading. This numerical result provides first the illustration of the numerical model compatibility with a new case of loading, and on a new kind of bone. Secondly, results of these studies (experimental and numerical), exemplify the importance of loading frequency in bone adaptation.

The parameter $a$, identified for two different bone materials (for a mouse ulna and a turkey ulna), leads to two different values, whose ratio is about 5 . Indeed, this parameter may depend on the microstructure of the bone. For the present cases, it implies that a larger fluid movement is required for the turkey case to get the same amount of gain in specific mass, when compared to the mouse case.

The sensitivity of the model to the reference parameter $\psi_{\text {ref }}$ is shown on Figures 4 and 5 ; though the error bars on the experimental data are related to dispersions, the error bars on numerical results have a different meaning: They correspond to different choices of values of $\psi_{\text {ref }}$ (without changing parameter a). Increasing $\psi_{\text {ref }}$ by $10 \%$ leads to an increase in bone remodeling for each frequency corresponding to the highest values of the bars. Conversely, a decrease of $-10 \%$ leads to the lower values for each loading frequency. On an average basis, this $\pm 10 \%$ relative variation on the sole parameter $\psi_{\text {ref }}$ leads to a $\pm 35 \%$ 
relative variation on the remodeling rate. On the other hand, the sensitivity to the parameter $a$ is given in Tables 3 and 5, where a sensitivity analysis is performed for the identification of this parameter. The relative variation of bone remodeling divided by the relative variation of $a$, on an average basis for both the studies, is $1.3 \% / \%$.

On the other hand, note that due to a lack of micro-mechanical interpretation of the parameter $a$, one has to care that both parameters $\psi_{\text {ref }}$ and $a$ should be identified on a same basis to get a consistent macroscopic model. For the same reason, though the phenomenological model is able to predict the experiments, it requires to be identified for each bone microstructure, which is a limitation of the model. The same limitation pertains for the 'Stanford' model.

\section{Conclusion}

This study proposes a predictive macroscopic model of bone remodeling which takes into account the effects of loading frequency and internal fluid movements. In a first step, a theoretical development introduces a daily stress stimulus of bone remodeling, which takes into account parameters of solid phase state (with stress) and fluid phase state (with Darcy velocity). In a second step, the daily stress stimulus is identified and validated using numerical simulations and experimental data.

Taking into account loading frequency via Darcy velocity, the numerical results agree with experimental data of [37], exemplifying privileged loading frequencies for bone remodeling between $5 \mathrm{~Hz}$ and $10 \mathrm{~Hz}$, for a significant force amplitude. Though not based on a micro-mechanical model of apposition and resorption, this predictable numerical model is able to simulate other kinds of loading, and other bone types, by identification of the parameter $a$. Once again, the numerical results agree with experimental data.

\section{References}

[1] D. Ambard and P. Swider. A predictive mechano-biological model of the bone-implant healing. European Journal of Mechanics. A/Solids, 25:927-937, 2006.

[2] S. Baïotto and M. Zidi. A study of the viscoelastic effect in a bone remodeling model. Biomechanics and Modeling in Mechanobiology, 8:129-139, 2009.

[3] G. S. Beaupré, T. E. Orr, and D. R. Carter. An approach for time-dependant bone modeling and remodeling - application: A preliminary remodeling simulation. Journal of Orthopaedic Research, 8:662-670, 1990.

[4] G. S. Beaupré, T. E. Orr, and D. R. Carter. An approach for time-dependent bone modeling and remodeling - theoretical development. Journal of Orthopaedic Research, 8:651-661, 1990.

[5] P. G. Coelho, P. R. Fernandes, H. C. Rodrigues, J. B. Cardoso, and J. M. Guedes. Numerical modeling of bone tissue adaptation - a hierarchical approach for bone apparent density and trabecular bone. Journal of Biomechanics, 42:830-837, 2009.

[6] O. Coussy. Mechanics of porous continua. John Wiley \& Sons, 1995.

[7] S. C. Cowin. Bone poroelasticity. Journal of Biomechanics, 32:217-238, 1999.

[8] S. C. Cowin. Mechanosensation and fluid transport in living bone. Journal of Musculoskeletal and Neuronal Interactions, 2(3):256-260, 2002.

[9] P. Fornells, J. M. Garcia-Aznar, and M. Doblaré. A finite element dual porosity approach to model deformation-induced fluid flow in cortical bone. Annals of Biomedical Engineering, 35(10):16871698, 2007.

[10] M. R. Forwood and C. H. Turner. Skeletal adaptations to mechanical usage: results from tibial loading studies in rats. Bone, 17:197S-205S, 1995.

[11] J. M. Garcia, M. Doblaré, and J. Cegonino. Bone remodelling simulation: a tool for implant design. Computational Materials Science, 25:100-114, 2002. 
[12] S. Gururaja, H. J. Kim, C. C. Swan, R. A. Brand, and R. S. Lakes. Modeling deformation-induced fluid flow in cortical bone's canalicular-lacunar system. Annals of Biomedical Engineering, 33(1):7$25,2005$.

[13] M. V. Hillsley and J. A. Frangos. Review: Bone tissue engineering: The role of interstitial fluid flow. Biotechnology and Bioengineering, 43:573-581, 1994.

[14] C. R. Jacobs, J. C. Simo, G. S. Beaupré, and D. S. Carter. Adaptative bone remodeling incorporating simultaneous density and anisotropy considerations. Journal of Biomechanics, 30:603-613, 1997.

[15] V. Kafka. On hydraulic strengthening of bones. Journal of Biomechanics, 26:761-762, 1993.

[16] R. G. Keanini, R. D. Roerf, and R. M. Dillaman. A theoretical model of circulatory interstitial fluid flow and species transport within porous cortical bone. Journal of Biomechanics, 28(8):901-914, 1995.

[17] J. Li, H. Li, L. Shi, A. S. L. Fok, C. Ucer, H. Devlin, K. Horner, and N. Silikas. A mathematical model for simulating the bone remodeling process under mechanical stimulus. Dental Materials, 23:1073-1078, 2007.

[18] E. Malachanne, D. Dureisseix, P. Canadas, and F. Jourdan. Experimental and numerical identification of cortical bone permeability. Journal of Biomechanics, 41:721-725, 2008.

[19] E. Malachanne, F. Jourdan, and D. Dureisseix. Numerical model of bone remodeling taking into account fluid phase. In Proceedings of International Conference on Tissue Engineering, pages 281288, Polytechnic Institute of Leiria, Portugal, July 2009.

[20] R. B. Martin. Porosity and specific surface of bone. CRC Critical Reviews in Biomedical Engineering, 10:179-222, 1984.

[21] Y. Matsuura, S. Oharu, T. Takata, and A. Tamura. Mathematical approaches to bone reformation phenomena and numerical simulations. Journal of Computational and Applied Mathematics, 158:107-119, 2003.

[22] J. G. McGarry, J. Klein-Nulend, M. G. Mullender, and P. J. Prendergast. A comparison of strain and fluid shear stress in stimulating bone cell responses - a computational and experimental study. The FASEB Journal, 19(3):482-484, 2005.

[23] M. Mengoni and J. P. Ponthot. Isotropic continuum damage/repair model for alveolar bone remodeling. Journal of Computational and Applied Mathematics, 234(7):2036-2045, 2010.

[24] D. Néron and D. Dureisseix. A computational strategy for thermo-poroelastic structures with a timespace interface coupling. International Journal for Numerical Methods in Engineering, 75(9):1053$1084,2007$.

[25] V.-H. Nguyen, T. Lemaire, and S. Naili. Poroelastic behaviour of cortical bone under harmonic axial loading: A finite element study at the osteonal scale. Medical Engineering and Physics, 32:384-390, 2010.

[26] A. M. Parfitt, C. H. E. Mathews, A. R. Villanueva, M. Kleerekoper, B. Frame, and D. S. Rao. Relationships between surface, volume, and thickness of iliac trabecular bone in aging and in osteoporosis. Implications for the microanatomic and cellular mechanisms of bone loss. Journal of Clinical Investigation, 72:1396-1409, 1983.

[27] Q.-H. Qin and J.-Q. Ye. Thermoelectroelastic solutions for internal bone remodeling under axial and transverse loads. International Journal of Solids and Structures, 41:2447-2460, 2004.

[28] Y.-X. Qin, T. Kaplan, A. Saldanha, and C. Rubin. Fluid pressure gradient, arising from oscillations in intramedullary pressure, is correlated with the formation of bone and inhibition of intracortical porosity. Journal of Biomechanics, 36:1427-1437, 2003.

[29] S. Ramtani and M. Zidi. Damaged-bone remodeling theory: Thermodynamical approach. Mechanics Research Communications, 26(6):701-708, 1999. 
[30] S. Ramtani and M. Zidi. A theoretical model of the effect of continuum damage on a bone adaptation model. Journal of Biomechanics, 34:471-479, 2001.

[31] A. G. Robling, F. M. Hinant, D. B. Burr, and C. H. Turner. Improved bone structure and strength after long-term mechanical loading is greatest if loading is separated into short bouts. Journal of Bone and Mineral Research, 17(8):1545-1554, 2002.

[32] G. Rouhi, M. Epstein, L. Sudak, and W. Herzog. Free surface density and microdamage in the bone remodeling equation: Theoretical considerations. International Journal of Engineering Science, 44:456-469, 2006.

[33] T. H. Smit, J. M. Huyghe, and S. C. Cowin. Estimation of the poroelastic parameters of cortical bone. Journal of Biomechanics, 35:829-835, 2002.

[34] A. E. Tami, P. Nasser, O. Verbogt, M. B. Schaffler, and M. L. Knothe Tate. The role of interstitial fluid flow in the remodeling response to fatigue loading. Journal of Bone and Mineral Research, 17(11):2030-2037, 2002.

[35] C. H. Turner. Three rules for bone adaptation to mechanical stimuli. Bone, 23:399-407, 1998.

[36] P. Verpeaux, A. Millard, T. Charras, and A. Combescure. A modern approach of large computer codes for structural analysis. In 10th International Conference on Structural Mechanics in Reactor Technology, Los Angeles, August 1989.

[37] S. J. Warden and C. H. Turner. Mechanotransduction in cortical bone is most efficient at loading frequencies of 5-10 Hz. Bone, 34:261-270, 2004.

[38] A. Watzky and S. Naili. Orthotropic bone remodeling: case of plane stresses. Mechanics Research Communications, 31:617-625, 2004.

[39] R. T. Whalen, D. R. Carter, and C. R. Steele. Influence of physical activity on the regulation of bone density. Journal of Biomechanics, 21:825-837, 1988.

[40] J. Wolff. Das Gesetz der Transformation der Knochen. Verlag von August Hirshwald, 1892. Translated by P. Maquet et R. Furlong, The Law of Bone Remodelling, Springer-Verlag, 1986.

[41] L. You, S. Temiyasathit, P. Lee, C. H. Kim, P. Tummala, W. Yao, W. Kingery, A. M. Malone, R. Y. Kwon, and C. R. Jacobs. Osteocytes as mechanosensors in the inhibition of bone resorption due to mechanical loading. Bone, 42:172-179, 2008. 